\title{
The Impact of Healthcare Traffic Over H2H and M2M Networks During the Spread Phase of Pandemic Diseases
}

\author{
K. Samhan*, A. H. EL Fawal**, M. Ammad-Uddin***, A. Mansour** and M. Najem** \\ *AUL University, Beirut, Lebanon \\ ** Lab-STICC, UMR 6285 - CNRS, ENSTA Bretagne, Brest, France \\ *** SNCS Research Centre, University of Tabuk, Kingdom of Saudi Arabia \\ *** Corresponding Author: mohammad.ammad@gmail.com
}

$\begin{array}{ll}\text { Submitted } & : 24 / 02 / 2021 \\ \text { Revised } & : 09 / 07 / 2021 \\ \text { Accepted } & : 11 / 10 / 2021\end{array}$

\begin{abstract}
Recently, the coronavirus pandemic has caused widespread panic around the world. Modern technologies can be used to monitor and control this highly contagious disease. A plausible solution is to equip each patient who is diagnosed with or suspected of having COVID-19 with sensors that can monitor various healthcare and location parameters and report them to the desired facility to control the spread of the disease. However, the simultaneous communication of numerous sensors installed in most of an area's population results in a massive burden on existing Long-Term Evolution (LTE) networks. The existing network becomes oversaturated because it has to manage two more kinds of traffic in addition to regular traffic (text, voice, and video). Healthcare traffic is generated by many sensors deployed over a huge population, and extra traffic is generated by people contacting their family members via video or voice calls. In pandemics, e-healthcare traffic is critical and should not suffer packet loss or latency due to network overload. In this research, we studied the performance of existing networks under various conditions and predicted the severity of network degradation in an emergency scenario. We proposed and evaluated three schemes (doubling bandwidth, combining LTE-A and LTE-M networks, and request queuing) for ensuring the quality of service (QoS) of healthcare sensor (HCS) network traffic without perturbation from routine human-to-human or machine-to-machine communications. We simulated all proposed schemes and compared them with existing network scenarios. Although it is observed from the results that doubling the bandwidth serves the purpose, it is a timeconsuming and expensive solution that seems non-practical in case a sudden peak occurs during an emergency. We can conclude by analyzing the simulations that the proposed queuing scheme is best-suitable under all studied scenarios where QoS for HCS traffic is never compromised, which is the ultimate goal of this research.
\end{abstract}

Keywords: Internet of Things (IoT); Long-Term Evolution Advanced (LTE-A); e-Healthcare.

\section{INTRODUCTION}

Modern history endorses many pandemics where massive numbers of people have been infected by highly contagious viruses, sometimes resulting in death. The most recent example is the coronavirus, which evolved rapidly and started threatening our lives. The coronavirus has been recorded in 235 countries with more than 600 million 
confirmed cases, according to the World Health Organization (WHO) (Coronavirus Disease (COVID-19) Pandemic, n.d.). In December 2019, several instances of possible pneumonia were recorded in Wuhan City of Hubei province in South China. In January 2020, the Chinese government and the WHO identified the causative infection as a novel coronavirus (SARS-CoV-2) belonging to the same infection group as the severe acute respiratory syndrome (SARS) that broke out in South China in 2002 and 2003. The coronavirus spread quickly across most locales in China after January 17, 2020, leading to more than 7,000 cases by the end of January (Zhong et al., 2020). The incidence continued to increase rapidly until the number of infected persons reached 11,500,302 cases on July 7, 2020. To date, the virus has no specific treatment or cure. Therefore, supportive therapy is considered a primary treatment for this disease to prevent negative effects on patients' health (Song et al., 2020). In this situation, the immediate course of action has proved most effective in preventing or slowing the spread of disease by restricting movement and tracking the physical contact of sick people.

The Internet of Things (IoT) is involved in every area of life, including home automation, smart meters, ehealthcare, and vehicle-to-vehicle communication. It is also best suited for monitoring, restricting, and managing infected people (Bajaj et al., n.d.) (Mehmood et al., 2017). Researchers at Harvard University and Massachusetts Institute of Technology have developed a smart tattoo ink capable of monitoring people's health (Powell \& University, n.d.). Many projects have focused on using IoT in healthcare facilities to monitor and control pandemics. However, installing sensors on all suspected and confirmed infected persons generates huge amounts of additional network traffic. In this scenario, communication of healthcare sensor (HCS) traffic may face severe challenges due to its coexistence with human-to-human (H2H )and machine-to-machine (M2M) traffic in terms of delayed access requests, network access latency, and data loss (Chen et al., 2018). Existing networks consist of a huge number of $\mathrm{H} 2 \mathrm{H}$ (e.g., video streaming, voice calls, gaming) and M2M (e.g., fire alarm sensors, electricity consumption sensors) transmission requests. Furthermore, adding massive HCS communication requests (e.g., heartbeat, oxygen, and body temperature sensors) may cause network overload and severely degrade the quality of service QoS of HCS traffic necessary to save human lives (Chen et al., 2018). Given these network issues, Harnessing IoT might lead to disaster in the health sector (Chen et al., 2018).

This paper simulates, compares, and analyzes various proposed solutions for handling this network overload problem. The purpose was to find a scheme capable of handling the emergency condition (i.e., the pandemic) with the existing network capacity. The main contribution of this research article is that we proposed a queueing model that can prioritize the communication of HCS devices (patient monitoring and tracking devices) over $\mathrm{H} 2 \mathrm{H}$ and M2M traffic in an emergency scenario such as the coronavirus pandemic. This proposed scheme maintains the QoS of HCS traffic by guaranteeing that $\mathrm{H} 2 \mathrm{H}$ and $\mathrm{M} 2 \mathrm{M}$ traffic do not affect $\mathrm{HCS}$ communication. We evaluated the proposed technique using simulation models, analyzed the results, and found that it performed well and successfully addressed the network issues observed in emergencies, such as network latency, starvation, and overload.

The rest of the paper is organized as follows. Section 2 describes the state of art. Section 3 discusses the proposed schemes for coping with the network bottleneck problem. Simulation modeling of the enhanced queuing method is provided in section 4, and the results are analyzed in section 5. Finally, a conclusion and suggested directions for future work are presented in section 6 .

\section{STATE OF THE ART}

The IoT is expanding in resources and entering new arenas, including the healthcare and medical fields. E-health technologies, including intelligent wearable sensors and smart applications, make healthcare facilities more effective and flexible. Therefore, a hospital can provide different healthcare IoT services through a wireless sensor network connected with IoT nodes, laboratory servers, and physicians (Chen et al., 2018). However, these services need to 
collect real-time patient data at each stage during their inpatient hospital stays, self-quarantine at home, consultancy at outpatients, and moving with care in society to monitor and analyze their health for a better life.

The medical field deals with a disease in three stages prevention response, early diagnosis, and quick treatment. Real-time data received from all the individuals diagnosed with, suspected of, and considered at high risk of revealing a disease helps at each stage to manage it more precisely and control it if it starts spreading. This kind of service generates high access demands of wireless networks, as every sensor reading collected from an individual may be critical for his health and safety. In addition, this collected data must be transmitted to the network either periodically or in real-time, but the important thing is it should be complete without delay or loss to prevent mortality. Furthermore, the life of IoT sensor nodes is also critical for more prolonged operation. The authors (Chen et al., 2018) proposed reduced-size transmission packets of communication links between sensor nodes to decrease power utilization.

Another research (Bai et al., 2020) proposed an intelligent diagnosis and treatment assistant program (nCapp) for the COVID-19 virus. nCapp is a software that provides questionnaires that need to answer by potential patients. This kind of assessment helps clinicians identify and isolate individuals suspected of having COVID-19. Therefore, the software assists in preventing the spread of the virus and contributes to the quality control of healthcare services during the COVID-19 pandemic, especially for outpatients.

The authors (Ullah et al., 2019) focused on the importance of implementing mobile health applications or IoT healthcare services using capabilities of $5 \mathrm{G}$ communication. 5G communication supports a large number of sensor devices, long battery life, enormous bandwidth, high network capability, very low latency, reliability, and security. However, according to the Global System for Mobile Communications Association (5G Global Launches \& Statistics), the 5G network will cover only one-third of the world's population by 2025, which is yet not fully implemented in most areas of the world. Moreover, the pandemic has further slowed the deployment and implementation of $5 \mathrm{G}$ due to the economic crisis and mobility restrictions. In addition, 5G-enabled devices are typically costly or not readily available, and it is often impossible to shift from $4 \mathrm{G}$ to $5 \mathrm{G}$ technologies in a short period of time. Therefore, more widely used cellular networks such as $4 \mathrm{G}$ and LTE need to provide higher bandwidth and low access latency to accommodate HCS devices without major network degradation.

The authors (Harb et al., 2020) proposed a new model that uses a stability-based $k$-means algorithm to analyze and train the system to predict and diagnose corona patients. This model is based on data collected from real-time health sensors (e.g., heart rate, body temperature). This study's proposed model's architecture consists of four layers:

1. Real-time monitoring of patient health;

2. Real-time decision-making and data storage;

3. Patient classification and disease diagnosis;

4. Data retrieval and visualization.

Moreover, each layer has a data mining algorithm to find correlations between patients' health parameters and the disease to classify and predict infected individuals.

As mentioned earlier in e-healthcare, the most critical task is to keep monitoring and collecting up-to-date data from the patient, either periodically or in real-time. Therefore, a low-cost fog-based monitoring system is proposed to monitor the patient's health remotely (Mutlag et al., 2019). In addition, Fog computing has the advantage of having intelligent gateways and IoT sensors. Furthermore, the IoT healthcare gadgets (such as body temperature, respiratory rate, heartbeat measurement, and oximeters) collect vital signs and send them wirelessly through gateways to the base station of the healthcare facilities to analyze them and assess the health of the patient. 
The authors (Outay et al., 2020) have worked and proposed a solution for handling one request per time slot. Therefore, the authors have proposed a methodology for $\mathrm{V} 2 \mathrm{~V}$ communication that will not be affected by $\mathrm{H} 2 \mathrm{H}$ and M2M devices using Adaptive eNode-B. Consequently, they offered to adapt the bandwidth according to the need until it reached its maximum capacity.

\section{PROPOSED ENHANCED QUEUE MODELING FOR HEALTHCARE TRAFFIC}

We propose a queue strategy that handles $\mathrm{HCS}, \mathrm{H} 2 \mathrm{H}$, and M2M traffic using a priority queue when the system reaches its peak. Requests that are pushed to the queue will have a higher priority in the next round of the emergency storm. Initially, the LTE-A network was designed to fulfill and serve the needs of H2H services. However, M2M communication was later introduced via the IoTs, which is continuously increasing and degrading the performance of LTE-A networks. By 2025, approximately 75 billion IoT devices will be added to the system, which is expected to generate trillion bytes of M2M communications annually (Ikpehai et al., 2019). In light of this current and expected network traffic, experts have focused on developing 5G networks. In addition, the COVID-19 pandemic in past years has created more challenges for the healthcare industry. In any future COVID-like situations, there will be a need for more HCS devices to prevent the spread of the pandemic and avoid putting people in danger. According to Statista (Saeed \& Alouini, n.d.), e-healthcare has the potential to achieve $\$ 3.3$ trillion in revenue by 2025 . The addition of this vast number of M2M and HCS devices in the future cannot be completed without an efficient and reliable network.

In light of this, many researchers have studied the coexistence of $\mathrm{H} 2 \mathrm{H}, \mathrm{M} 2 \mathrm{M}$, and HCS communications in LTE-A networks. In this article, we mathematically characterize the key performance features of $\mathrm{H} 2 \mathrm{H}, \mathrm{M} 2 \mathrm{M}$, and HCS communications. Additionally, we propose an enhanced queue method using a continuous-time Markov chain (CTMC) model with the following objectives:

- Study the mutual impact of $\mathrm{H} 2 \mathrm{H}$ and M2M traffic on HCS communication.

- Measure, analyze, and identify HCS congestion problems.

- Model a new framework called enhanced queue modeling for HCS traffic in the LTE-A network.

Our system is an $\mathrm{M} / \mathrm{M} / 1$ queue model with one server, with the arrival of entities followed by the Poisson process and a service time with an exponential distribution. To evaluate the impact of $\mathrm{H} 2 \mathrm{H}$ and $\mathrm{M} 2 \mathrm{M}$ transmission in the emergency scenario for healthcare traffic, we assume an LTE-A network using a bandwidth of $1.4 \mathrm{MHz}(\mathrm{C}=$ 6 Resource Blocks) to stress the system maximally. The following are the parameters used to define the proposed system:

- $\lambda_{\mathrm{M} 2 \mathrm{M}}$ represents the arrival rate of an $\mathrm{M} 2 \mathrm{M}$ communication (direct communication between machines over wireless channels).

- $\lambda_{\mathrm{H} 2 \mathrm{H}}$ represents the arrival rate of pure $\mathrm{H} 2 \mathrm{H}$ communication (communication between humans through VoIP, file transfer, and so on).

- $\lambda_{\text {HCS }}$ represents the average arrival rate of pure healthcare communications (communication between HCSs and hospital servers or healthcare machines).

- $\quad \mu_{\mathrm{M} 2 \mathrm{M}}, \mu_{\mathrm{H} 2 \mathrm{H}}$, and $\mu_{\mathrm{HCS}}$ represent the service rate for each technology.

- $\lambda_{\mathrm{M} 2 \mathrm{M}}, \lambda_{\mathrm{H} 2 \mathrm{H}}$, and $\lambda_{\mathrm{HCS}}$ represent the average arrival rate of each traffic with the service rate of $\mu_{\mathrm{M} 2 \mathrm{M}}, \mu_{\mathrm{H} 2 \mathrm{H}}$, and $\mu_{\mathrm{HCS}}$, respectively.

- $\mathrm{H} 2 \mathrm{H}$ and M2M traffic are assigned the same priority, while HCS is given the highest.

- A priority queue type is used with a specified capacity size. 
In our previous study (El Fawal et al., 2018), we started receiving one request per time slot for each type of traffic (M2M and $\mathrm{H} 2 \mathrm{H})$ and observed no limitation problems, as expected. We then began to increase the number of requests, and ultimately server stopped accepting submissions at 52,000 to avoid overload. In this article, we upgrade our previous system. The new proposed system can simultaneously handle two requests per time slot of different traffic types, including $\mathrm{HCS}, \mathrm{M} 2 \mathrm{M}$, and $\mathrm{H} 2 \mathrm{H}$, even in a very saturated state.

The proposed CTMC analytical model consists of four steps:

1. Use of CTMC model: it is used as a stochastic process to designate the different states of every possible event for $\mathrm{H} 2 \mathrm{H}, \mathrm{M} 2 \mathrm{M}$, and HCS traffic.

2. Defining equilibrium equations: describing all transition probabilities from one state to another.

3. Defining a Linear system: Linear system is described by equilibrium equations.

4. Network evaluation: Finally, performance metrics are generated to characterize the performance of $\mathrm{H} 2 \mathrm{H}$, M2M, and HCS traffic.

The network traffic in our system consists of average arrival rates of $\mathrm{H} 2 \mathrm{H}, \mathrm{M} 2 \mathrm{M}$, and HCS requests (represented as $\lambda_{\mathrm{H} 2 \mathrm{H}}, \lambda_{\mathrm{M} 2 \mathrm{M}}$, and $\lambda_{\mathrm{HCS}}$, respectively). Each incoming request is represented by a state $S(i, j, m, n)$ where $i$ and $j$ represent the number of $\mathrm{H} 2 \mathrm{H}$ and $\mathrm{M} 2 \mathrm{M}$ requests served, respectively, while $i+j$ represents the ongoing $\mathrm{HCS}$ traffic. Similarly, the number of requests waiting in the queue is denoted by $m, n$, and $m+n$ for each type of traffic. The working queuing model or proposed system is shown in Figure 1.

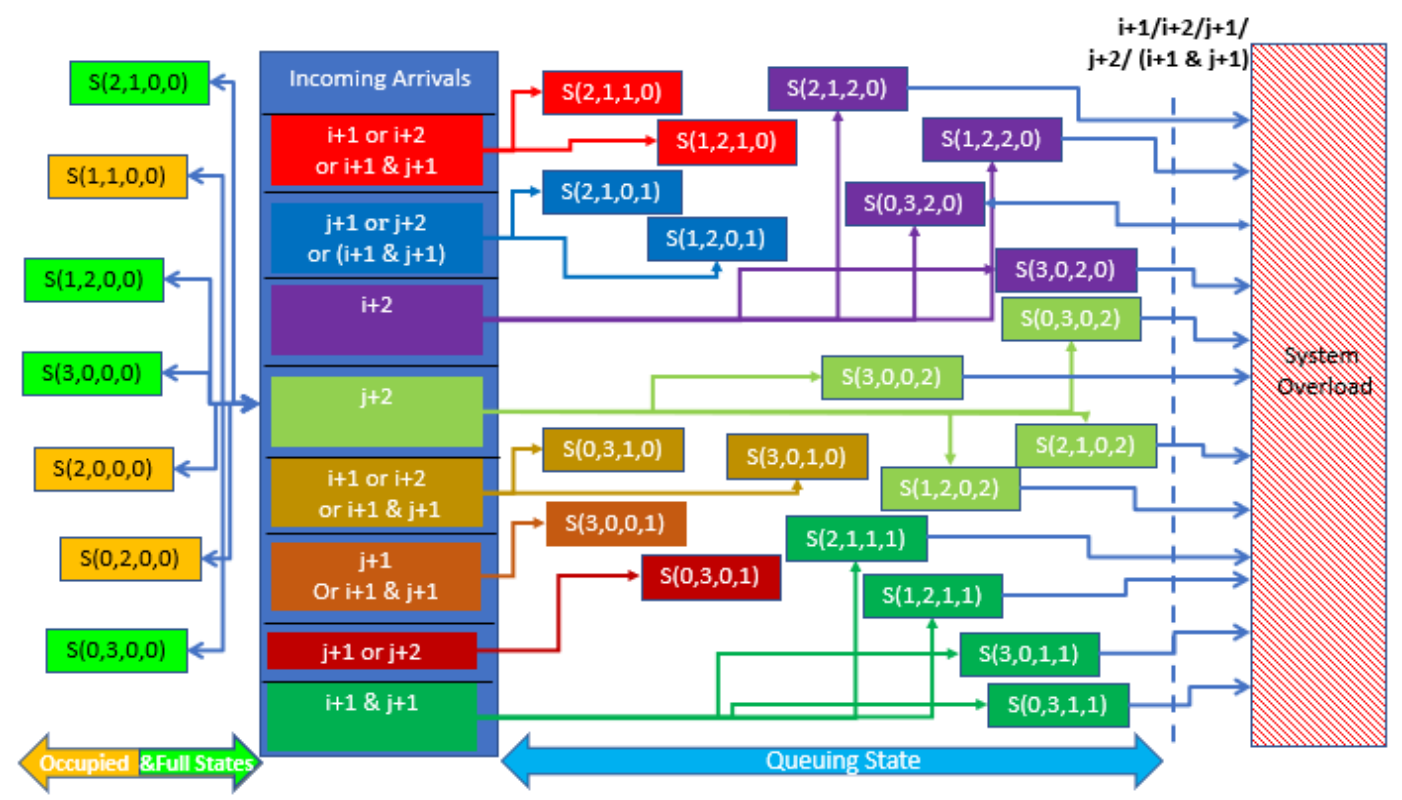

Figure 1. Proposed generic queue model representation for handling two requests per slot.

Figure 1 shows how the system handles incoming HCS, H2H, and M2M traffic and pushes such traffic to priority queues until the system reaches maximum capacity. Requests pushed to the queue are assigned higher priority to be served in the next round of the emergency storm. The system's functioning is explained through an example expressing the states of every request in Figure 2. 


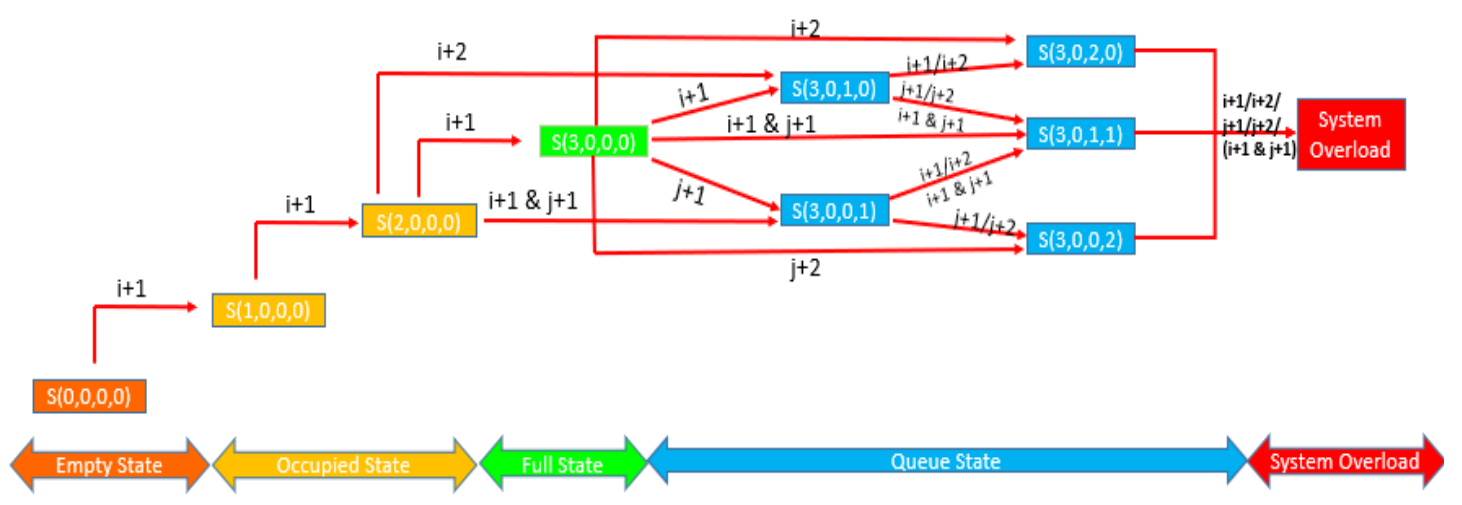

Figure 2. Simple CTMC states being pushed to queue states, where $S$ represents a state.

When a new request arrives, the system checks whether the LTE-A network is fully reserved. If yes, the request is pushed to the queue; if not, the system allocates a resource block for it. The entire network is reserved for all arrived packets if the queue is empty. The request will be sent to the network for resource allocation if it is partially empty. Ultimately, if the queue becomes full (meaning that the system is full), the request will be rejected until a free resource is available (i.e., there is room in the queue), as shown in the flow chart in Figure 3.

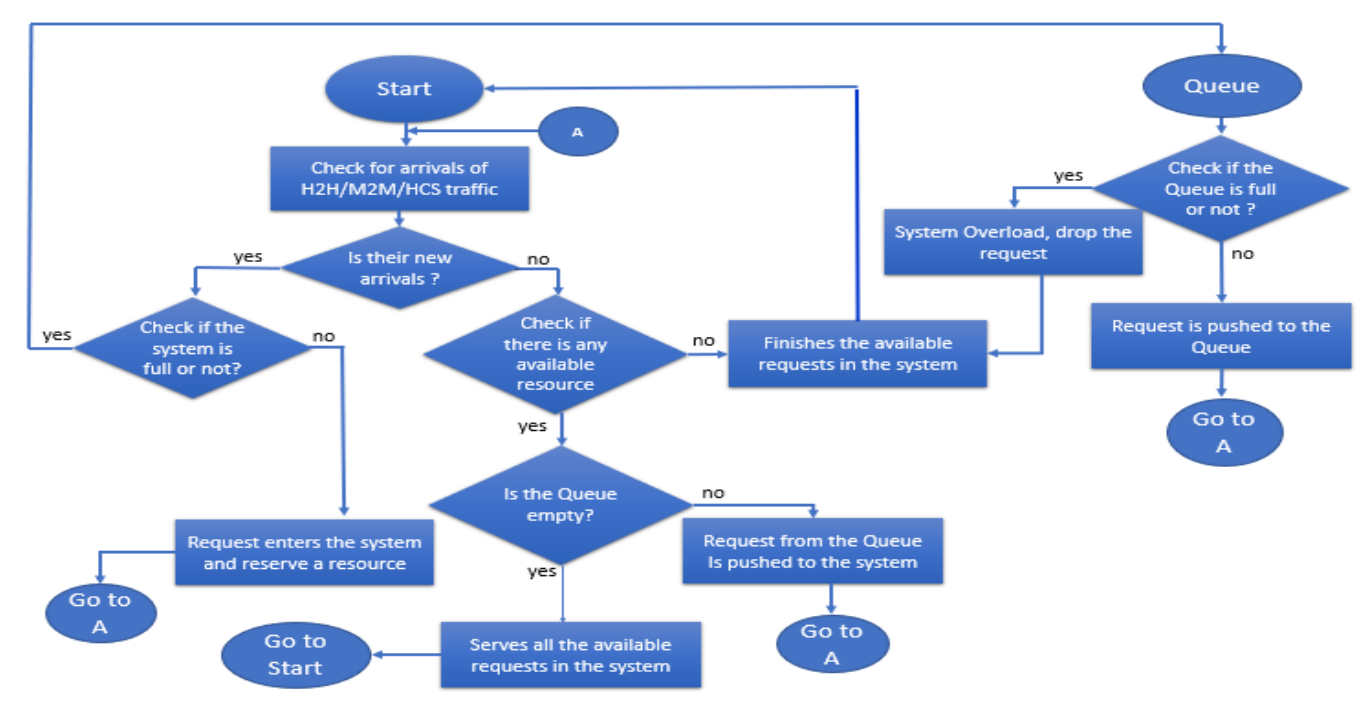

Figure 3. Flow chart of queue model.

\section{A. Equilibrium Equations}

The equilibrium equations represent that the requests arrive with an average rate of $\lambda$ while their service rate is $\mu$. Thus, the equilibrium equation can be created according to Markov Chain (El Fawal et al., 2018). The equilibrium equations for each state (empty, occupied, full, and queue) of the serving queue are as follows: 


\section{Empty State:}

$\left(2 \lambda_{1}+2 \lambda_{2}+\left(\lambda_{3}\right) * \Pi(0,0,0,0)=\left(\mu_{1} * \Pi(1,0,0,0)\right)+\left(\mu_{2} * \Pi(0,1,0,0)\right)+\left(\mu_{1} * \Pi(2,0,0,0)\right)+\left(\mu_{2} * \Pi(0,2,0,0)\right)+\right.$ $\left(\left(\mu_{3}\right) * \Pi(1,1,0,0)\right)$

\section{Occupied State:}

$\left(\lambda_{1} * \Pi(0,0,0,0)\right)+\left(\mu_{1} * \Pi(2,0,0,0)\right)+\left(\mu_{2} * \Pi(1,1,0,0)\right)+\left(\mu_{1} * \Pi(3,0,0,0)\right)+\left(\mu_{2} * \Pi(1,2,0,0)\right)+\left(\left(\mu_{3}\right) * \Pi(2,1,0,0)\right)$ $=\left(2 \lambda_{1}+2 \lambda_{2}+\left(\lambda_{3}\right)+\mu_{1}\right) * \Pi(1,0,0,0)$

\section{Full State:}

$\left(\lambda_{1} * \Pi(0,2,0,0)\right)+\left(\lambda_{2} * \Pi(1,1,0,0)\right)+\left(\lambda_{2} * \Pi(1,0,0,0)\right)+\left(\left(\lambda_{3}\right) * \Pi(0,1,0,0)+\mu_{2} * \Pi(1,2,0,1)+\mu_{1} * \Pi(1,2,1,0)+\mu_{1}\right.$

$\left.* \Pi(1,2,2,0)+\mu_{2} * \Pi(1,2,0,2)+\mu_{3} * \Pi(1,2,1,1)\right)=\left(\left(\mu_{1}+2 \mu_{2}+\left(\mu_{3}\right)+2 \lambda_{1}+2 \lambda_{2}+\lambda_{3}\right) * \Pi(1,2,0,0)\right)$

\section{Queue State:}

$\lambda_{1} * \Pi(1,2,0,0)+\lambda_{3} * \Pi(1,1,0,0)+\lambda_{1} * \Pi(0,2,0,0)=\left(2 \mu_{1}+\mu_{3}\right) * \Pi(1,2,1,0)$

Where $\lambda_{1}, \lambda_{2}$, and $\lambda_{3}$ represent the respective average arrival rates for M2M, H2H, and HCS traffic. While $\mu_{1}, \mu_{2}$, and $\mu_{3}$ represent their average service rates; and $\Pi$ represents the probability of every state.

As described above, $i, j, m$, and $n$ denote the number of ongoing services of $\mathrm{H} 2 \mathrm{H}, \mathrm{M} 2 \mathrm{M}$, and HCS traffic. When a service is achieved, the system moves from one state to another, or a new request arrives (by increasing or decreasing $i$ or $j$ ) with a steady-state probability $\pi(i, j)$. We can derive a linear equation as follows:

$\sum_{i=0}^{C} \sum_{j=0}^{C-1} \pi(i, j)=1,0 \leq \pi(i, j) \leq 1$

The steady-state probability vector $\pi$ is represented in a square matrix as

$$
\pi=\left(\begin{array}{c}
\pi(0,0) \\
\pi(0,1) \\
\cdot \\
\pi(c, 0)
\end{array}\right)
$$

where $\mathrm{C}$ represents the number of resource blocks.

\section{B. Service Completion Rate}

A square matrix called Service Completion Rate (SCR) is extracted To validate the proposed model.

$S C R=\sum_{i, j} i \mu \pi(i, j)$ 
MATLAB is used to solve the linear system as per equation (1), and SCR of the equilibrium equation is calculated as per equation (3) to validate our system mathematically. The Simulation models have also been generated accordingly.

\section{Validating Our Model with Mathematical Equations}

A mathematical model is derived using Markov Chain to validate our Simulink model. For simplicity, we are going to assume that the arrival rate for $\mathrm{M} 2 \mathrm{M}, \mathrm{HCS}$, and $\mathrm{H} 2 \mathrm{H}$ is equal to 4 , represented by $(\lambda \mathrm{M} 2 \mathrm{M}=4),(\lambda \mathrm{HCS}=4)$, and $(\lambda \mathrm{H} 2 \mathrm{H}=4)$ respectively. Where $\mathrm{C}=6$ and their service rate equal to $1(\mu \mathrm{H} 2 \mathrm{H}=1, \mu \mathrm{M} 2 \mathrm{M}=1 \mu \mathrm{HCS}=1)$. The system is implemented in MATLAB using Markov Chain and observed the acceptance of each traffic as follows:

$$
\text { SCR_M } 2 \mathrm{M}=100 \%, \mathrm{SCR} \_\mathrm{H} 2 \mathrm{H}=50 \% \text { and SCR_HCS }=0 \%
$$

Since, in MATLAB, we cannot set up priority, the requests are served as per First Input First Output (FIFO). Therefore, in Simulink, we simulate the system by giving the same parameters in addition to priorities. The highest priority is given to M2M traffic, then comes $\mathrm{H} 2 \mathrm{H}$, and finally HCS, and the result is shown in Figure 4.
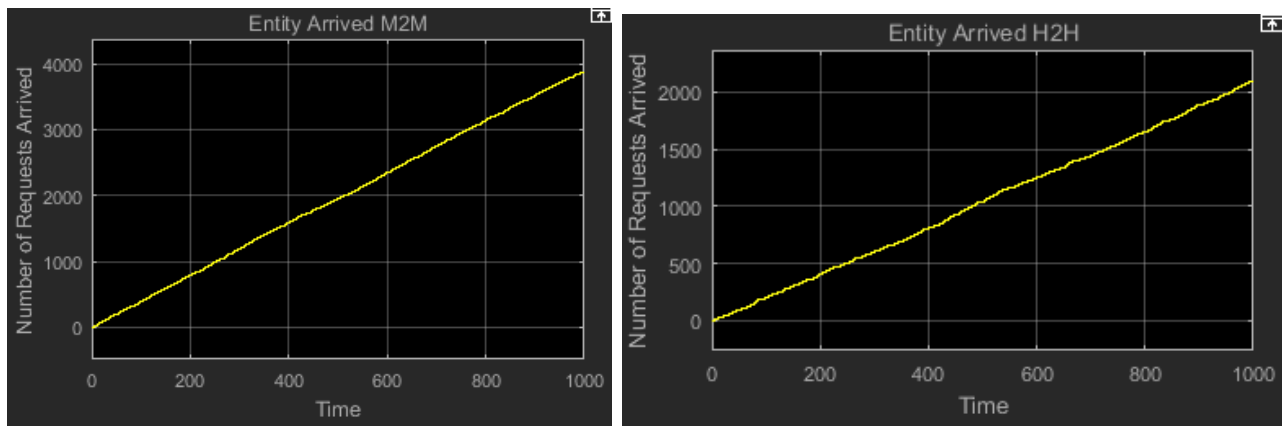

Figure 4. Entity Arrived of $\mathrm{H} 2 \mathrm{H}$ and $\mathrm{M} 2 \mathrm{M}$ for Validation Scenario.

According to the results shown in Figure 4, 2000 requests of $\mathrm{H} 2 \mathrm{H}$ are served out of 4000 , where $\lambda \mathrm{H} 2 \mathrm{H}=4$. While all 4000 requests of $\mathrm{M} 2 \mathrm{M}$ are served where $\lambda \mathrm{M} 2 \mathrm{M}=4$. Therefore, we can conclude that our system is working properly and validated.

So, to analyze the impact of $\mathrm{H} 2 \mathrm{H}$ and $\mathrm{M} 2 \mathrm{M}$ over $\mathrm{HCS}$ traffics, we are going to simulate different scenarios.

\section{NETWORK SIMULATION FOR PERFORMANCE ANALYSIS}

The proposed system was evaluated using a simulation study. Different models were created using Markov chain methods to describe system variations. The OMNeT++ framework and MATLAB Simulink environment is used to create different simulation scenarios. Different simulation runs were developed for each scenario to test each possible network situation. Four simulation scenarios were created to represent four types of network settings:

1. Existing network;

2. The network was upgraded by doubling bandwidth;

3. Combination of LTE-A and LTE-M networks;

4. Network with the proposed queuing scheme. 
The performance of each network model was evaluated in both normal and emergency conditions. Different simulation runs were created for each network model as follows:

1. Less populated area;

2. Highly populated area;

3. Low-frequency emergency situation $\left(\lambda_{\mathrm{HCS}}=1\right.$ request $)$;

4. $\quad$ Moderate-frequency emergency situation $\left(\lambda_{\mathrm{HCS}}=4\right.$ requests);

5. High-frequency emergency situation $\left(\lambda_{\mathrm{HCS}}=13\right.$ requests).

A hierarchical representation of simulation runs is shown in Figure 5.

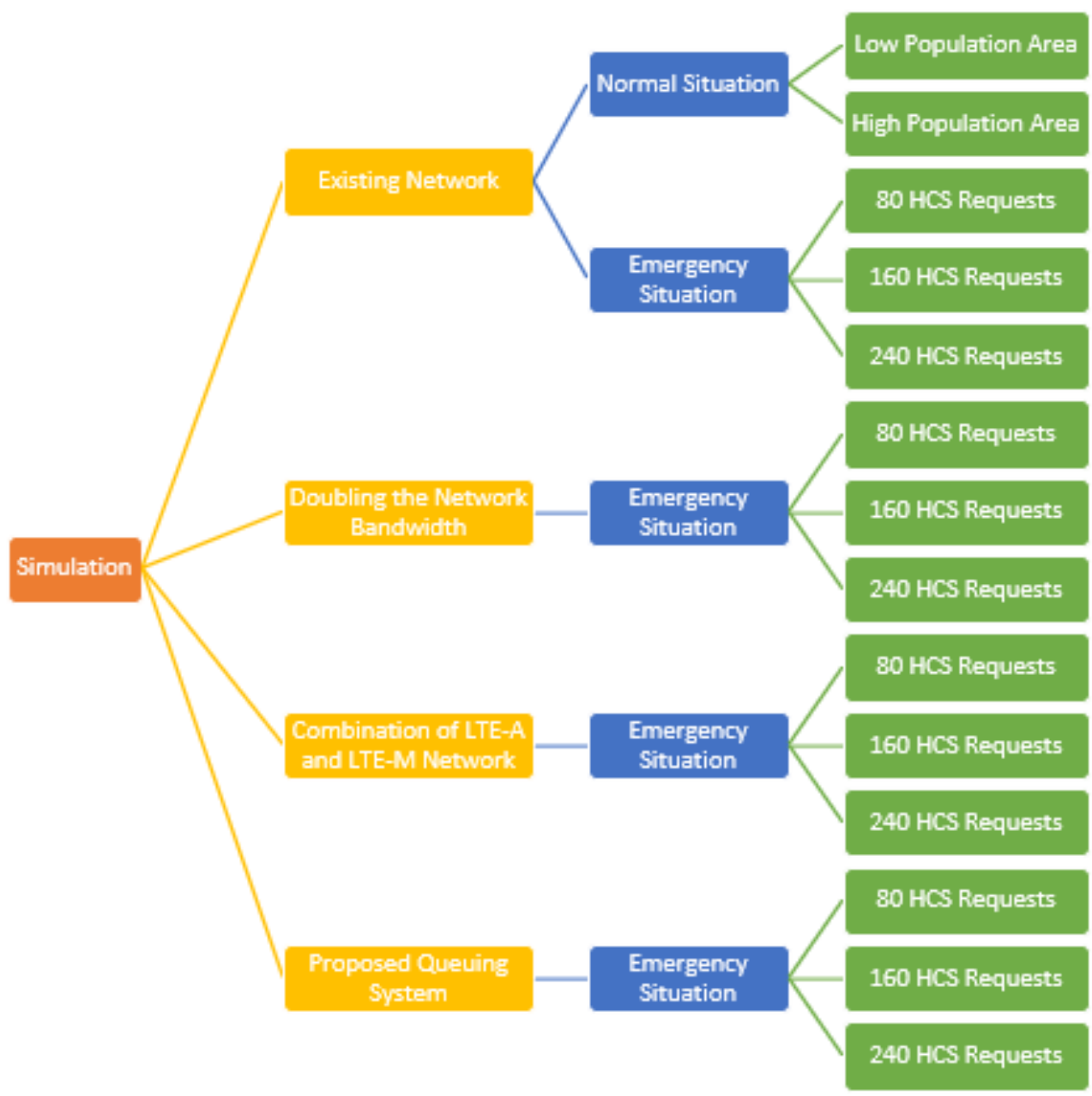

Figure 5. Simulation models.

We developed a city communication network in $\mathrm{OMNeT}++$, where we generated $\mathrm{H} 2 \mathrm{H}, \mathrm{M} 2 \mathrm{M}$, and $\mathrm{HCS}$ traffic routed through different servers, as shown in Figure 6. 


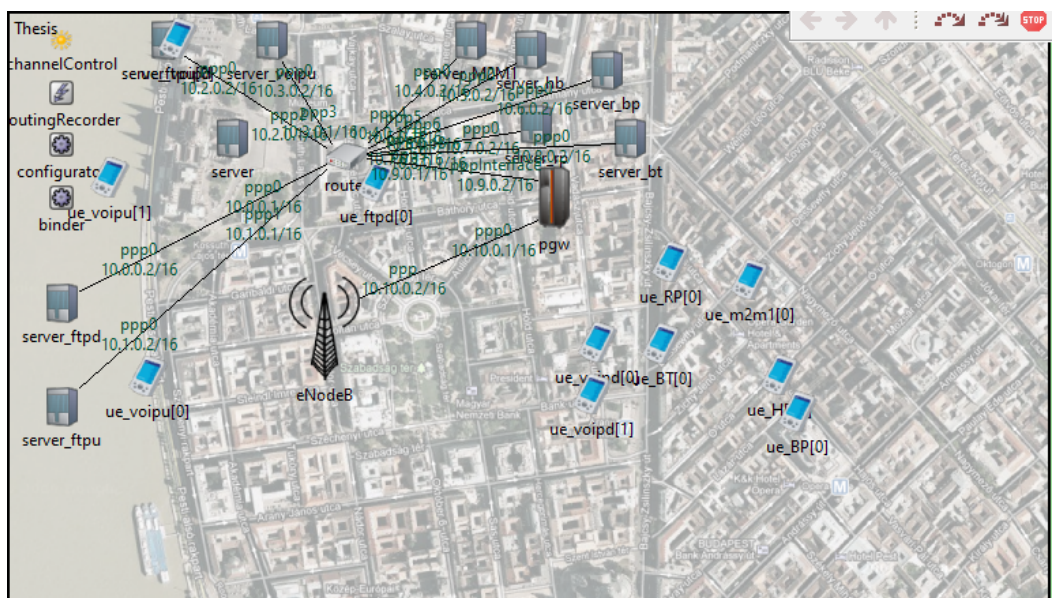

Figure 6.: SimuLTE scenario representing LTE-A network.

Using the network generated in OMNeT++, we designed many scenarios, shown in Figure 4, to evaluate the performance of the LTE-A network when handling different types of traffic and the impact of HCS traffic on $\mathrm{H} 2 \mathrm{H}$ and M2M transmission and vice versa. The SimuLTE parameters for the LTE-A network settings are shown in Table 1.

Table 1. Simulation parameters.

\begin{tabular}{|c|c|}
\hline Parameter & Value \\
\hline Simulation length & $200 \mathrm{~s}$ \\
\hline Terminal velocity & $120 \mathrm{~km} / \mathrm{s}$ \\
\hline Mobility type & Linear mobility \\
\hline Transmission bandwidth & $1.4 \mathrm{MHz}$ (for download and upload each) \\
\hline Number of PRBs & 6 (for download and upload each) \\
\hline
\end{tabular}

\section{A. Simulation Model of Existing Network}

We evaluated the performance of the existing network under different conditions, including normal versus emergency conditions and less populated versus highly populated areas. First, the performance of the server was observed by setting $\mathrm{H} 2 \mathrm{H}$ traffic at the highest priority and M2M and HCS at the same priority, with the following parameters:

- An arrival rate of $\mathrm{H} 2 \mathrm{H}$ requests such that $\lambda_{\mathrm{H} 2 \mathrm{H}}=1$;

- An arrival rate of $\mathrm{M} 2 \mathrm{M}$ requests such that $\lambda_{\mathrm{M} 2 \mathrm{M}}=1$;

- An arrival rate of $\mathrm{HCS}$ requests such that $\lambda_{\mathrm{HCS}}=2$;

- $\mu_{\mathrm{H} 2 \mathrm{H}}=0.5, \mu_{\mathrm{M} 2 \mathrm{M}}=1$, and $\mu_{\mathrm{HCS}}=1$. 
The results of at least three runs were averaged to calculate the SCR of the system, and traffic was analyzed. The results show that all traffic was fully served in the LTE-A network with a bandwidth of $1.4 \mathrm{MHz}$ and 6 PRBs. These results show that the system is working normally since the devices only occupy an average of five out of six resources. Since $\mathrm{H} 2 \mathrm{H}$ traffic is generated with an arrival rate of $\mu_{\mathrm{H} 2 \mathrm{H}}=0.5$, it reserves one out of six resources for two consecutive intervals. This means that the system does not suffer from any overload. Hence, the server utilization index of the system is as follows:

$\mathrm{SUI}=($ sum of the number of arrival rate $/$ total number of resource blocks $)=0.83$.

The observed server utilization of the system is approximately $80 \%$, which means the system is relaxed and working freely without congestion. We tested this model for both highly and less inhabited areas and found it works well in both. The results are shown in Figures 7 and 8.

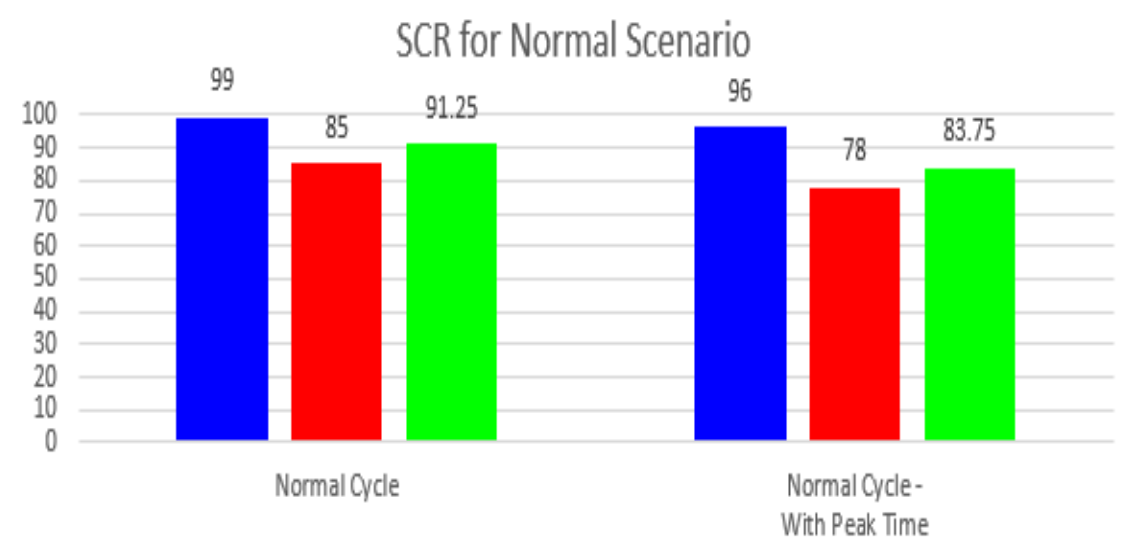

- $\mathrm{H} 2 \mathrm{H} \square \mathrm{M} 2 \mathrm{M}=\mathrm{HCS}$

Figure 7. Performance of existing network in less inhabited areas.

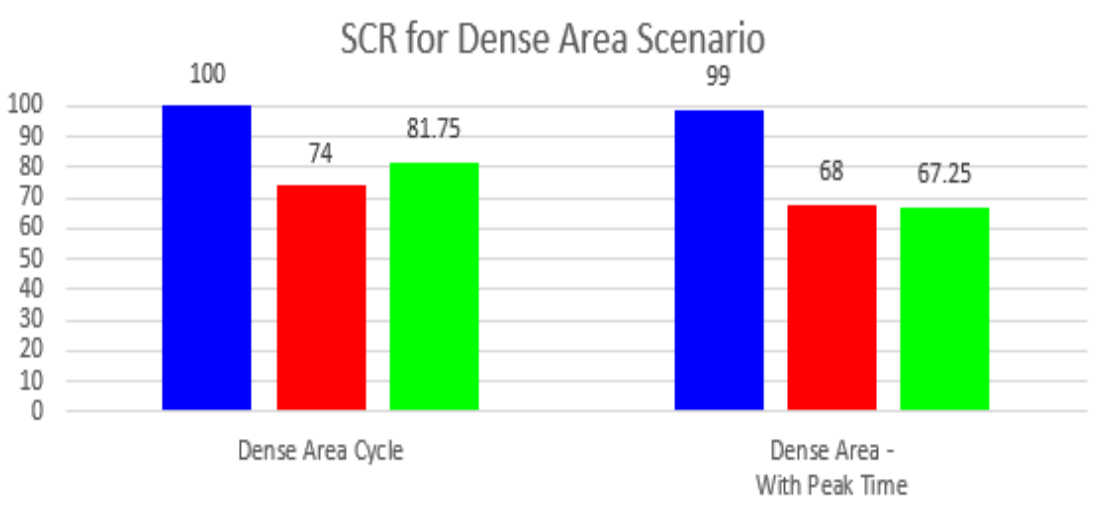

- $\mathrm{H} 2 \mathrm{H} \square \mathrm{M} 2 \mathrm{M} \| \mathrm{HCS}$

Figure 8. Performance of existing network in highly inhabited areas. 


\section{B. Simulation Model of Existing Network in Emergency Conditions}

We also evaluated the performance of the existing network in emergency conditions. In an emergency scenario, the number of e-HCSs is expected to increase enormously. In this situation, we focused on analyzing and observing the performance of the LTE-A network for patient devices (e.g., heartbeat, blood pressure, respiratory, and body temperature sensors). We considered three parameters for the emergency scenario as follows:

1. The emergency scenario considered $20 \mathrm{H} 2 \mathrm{H}$ users (including VoIP-DL and VoIP-UL), $10 \mathrm{M} 2 \mathrm{M}$ users, and 80 HCSs (including heartbeat, body temperature, and respiratory sensors) with six PRBs.

2. The emergency scenario with moderate traffic load time considered $20 \mathrm{H} 2 \mathrm{H}$ users, and10 M2M users, and 160 HCSs with six PRBs.

3. The emergency scenario with full peak time considered $20 \mathrm{H} 2 \mathrm{H}, 10 \mathrm{M} 2 \mathrm{M}$ users, and $240 \mathrm{HCSs}$ with six PRBs.

After simulating the above cases, the results showed that the $\mathrm{H} 2 \mathrm{H}$ traffic revealed no changes. VoIP traffic has a higher priority than M2M and e-health traffic when accessing the network in normal situations. Moreover, as the number of e-HCSs increases, M2M devices suffer from performance degradation due to low priority. However, eHCSs suffer from severe performance degradation (bandwidth starvation) due to the overload of the LTE-A network caused by the huge number of devices attempting to access the network, as shown in Figure 9. Moreover, M2M performance is maintained at the same level, but HCS dropped by up to $48 \%$, which is unacceptable, given that it is a matter of human life.

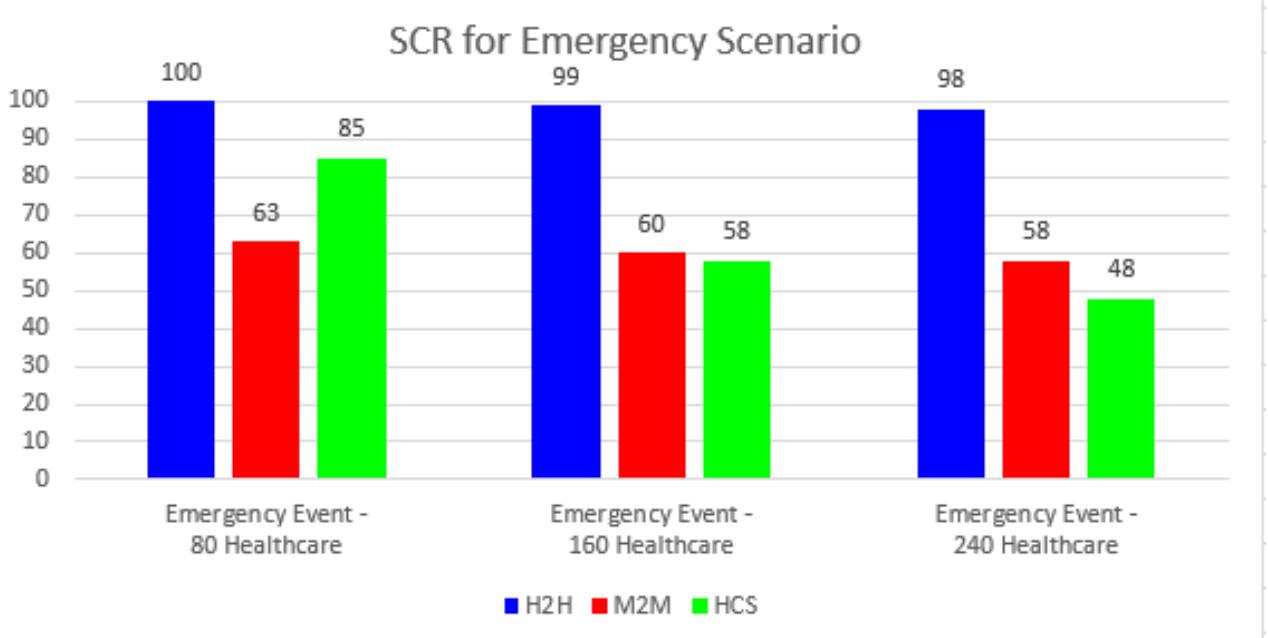

Figure 9. Performance of existing network under varying emergency conditions.

The extreme emergency scenario was tested by increasing the number of HCS devices to as much as 13,000. We observed that the SCR of HCS and M2M traffic dropped to 20\%, while H2H communication maintained its QoS at the cost of HCS and M2M service. It is not acceptable, especially when fighting a pandemic like COVID-19. 


\section{Emergency Scenario of an Existing Network}

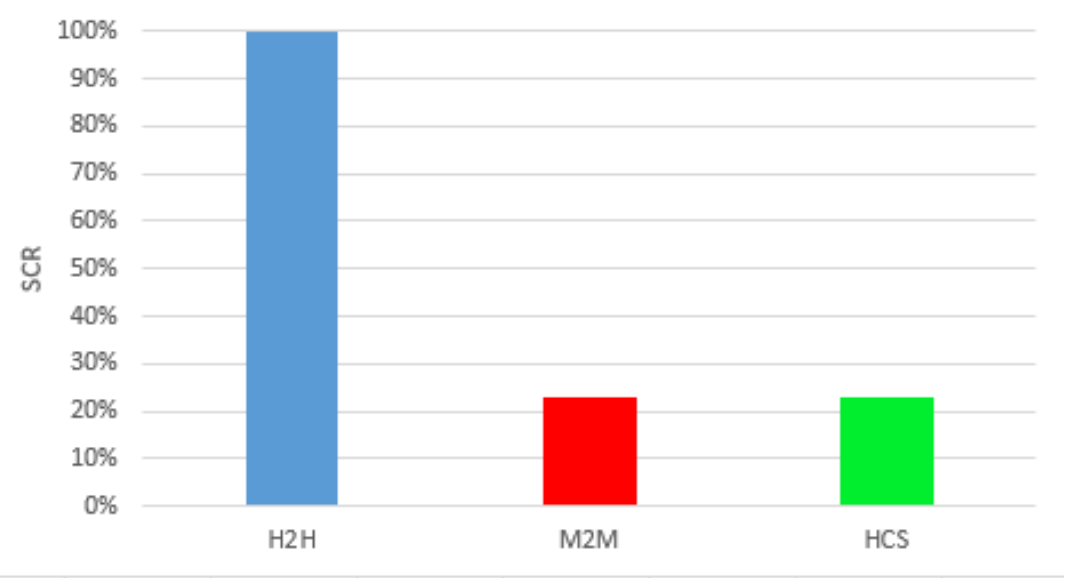

Figure 10. The results of the simulation model of an existing network under extreme emergency conditions.

\section{Simulation Model of Existing Network Upgraded by Doubling Bandwidth}

In this model, we evaluated the upgraded existing network. The network was upgraded by increasing the bandwidth to $400 \%$. We will test how it will work under emergency condition.

In this simulation case, all the parameters were the same as those in the previous emergency scenario but with 25 PRBs to solve the congestion issue. The performance of LTE-A network is improved in handling e-HCSs, as shown in Figure 9. The results of the SCR show that the $\mathrm{H} 2 \mathrm{H}$ traffic maintained $100 \%$ performance due to its high priority level. In contrast, the SCR of e-HCSs improved until it reached approximately $80 \%$ as the number of e-HCSs increased (in contrast to $48 \%$ with six PRBs). This means that e-HCSs suffered from neither access delay nor overload. The results are shown in Figure 11.

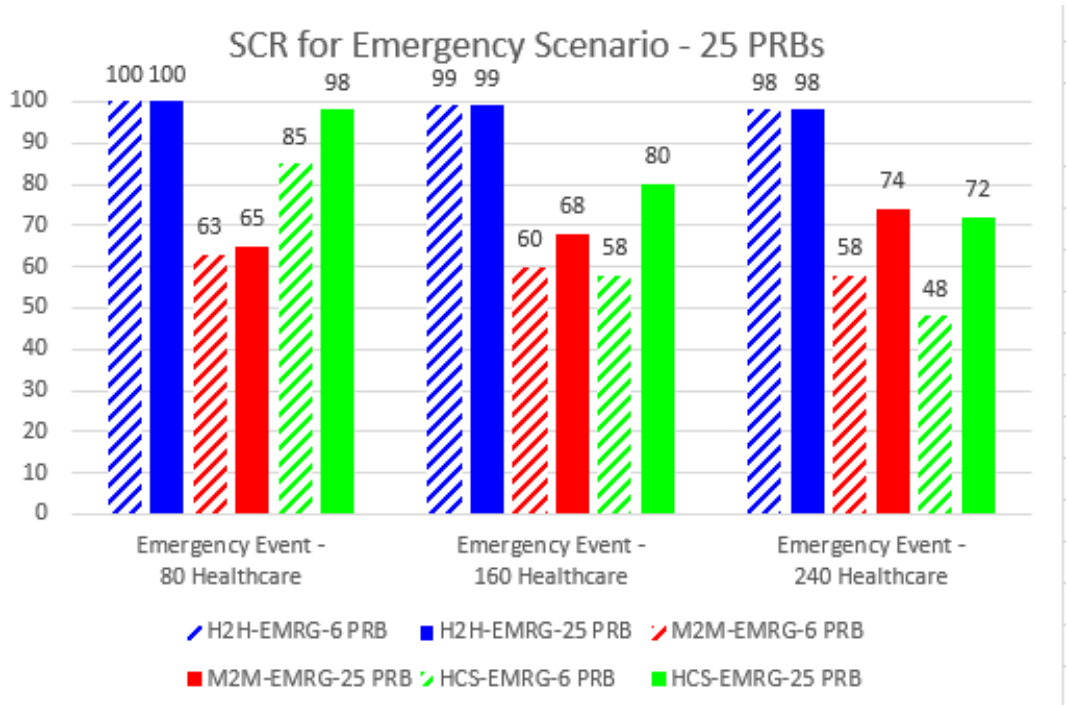

Figure 11. Performance of upgraded existing network under emergency conditions. 


\section{Simulation Model of Combined LTE-A and LTE-M Network}

In this simulation run, we tested the performance of a network system with coexisting LTE-A and LTE-M networks to solve the overload and access delay problems. In this simulation, we assigned H2H traffic to the LTE-A network and M2M and e-healthcare traffic to the LTE-M network. It ensured that the high priority of H2H traffic while accessing the network would not affect the performance of e-healthcare traffic, as shown in Figure 12. SCR for emergency scenario with LTE-A and LTE-M networkFigure 12. The results show that e-HCSs performance is improved until it reaches around $70 \%$ as the number of e-HCSs increases. It means that $\mathrm{H} 2 \mathrm{H}$ traffic does not affect e-healthcare traffic when accessing the network.

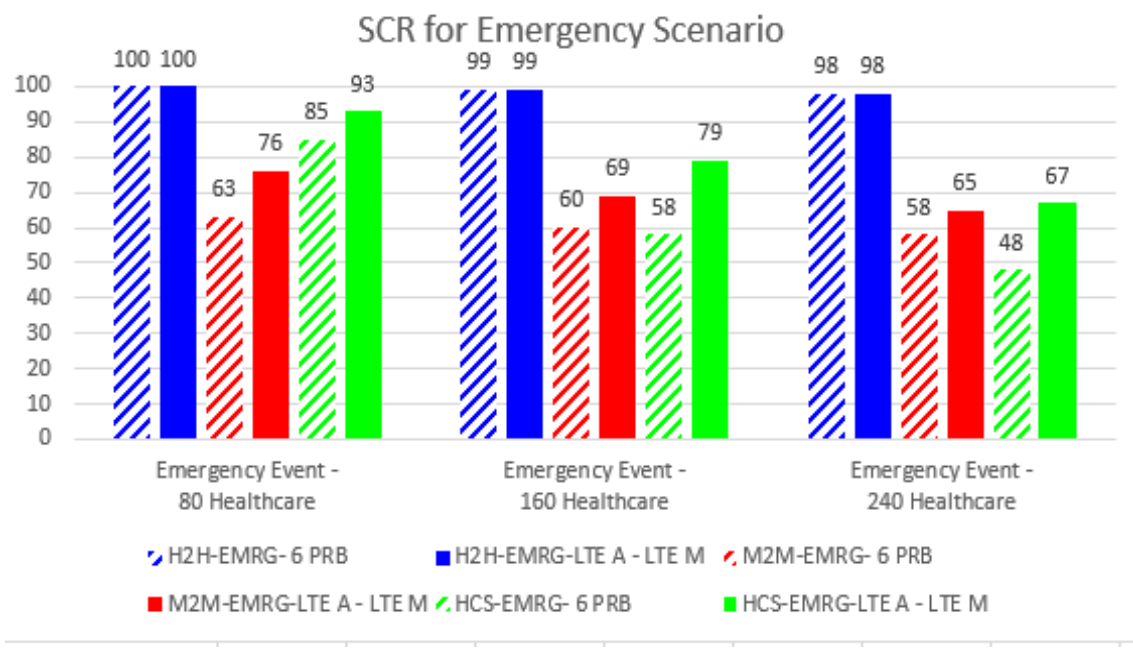

Figure 12. SCR for emergency scenario with LTE-A and LTE-M networks.

\section{E. Simulation Model for Proposed Queuing Scheme}

In this scenario, we gave the highest priority to queue traffic, HCS traffic with the second-highest priority, and $\mathrm{H} 2 \mathrm{H}$ and M2M traffic with the joint third-highest priority. We assume two waves of normal traffic with a queue capacity of five.

\section{First Storm for Emergency Scenario Using Queue Strategy}

We assigned the highest priority to queue traffic, HCS traffic with the second-highest priority, and similar priorities for $\mathrm{H} 2 \mathrm{H}$ and $\mathrm{M} 2 \mathrm{M}$ traffic. We assumed two waves for normal traffic, with a queue capacity of five. In the first wave, the queue was set at zero since, initially, the queue is empty. Traffic was generated for the simulation according to the following parameters:

- An arrival rate of $\mathrm{H} 2 \mathrm{H}$ requests such that $\lambda_{\mathrm{H} 2 \mathrm{H}}=1$;

- $\quad$ An arrival rate of $\mathrm{M} 2 \mathrm{M}$ requests such that $\lambda_{\mathrm{M} 2 \mathrm{M}}=2$;

- An arrival rate of HCS requests such that $\lambda_{\mathrm{HCS}}=4$;

- An arrival rate of queue requests such that $\lambda_{\text {Queue }}=0$;

- $\quad{ }_{\mu \mathrm{H} 2 \mathrm{H}}=0.5, \mu_{\mathrm{M} 2 \mathrm{M}}=1, \mu_{\mathrm{HCS}}=1$, and $\mu_{\text {Queue }}=1$. 


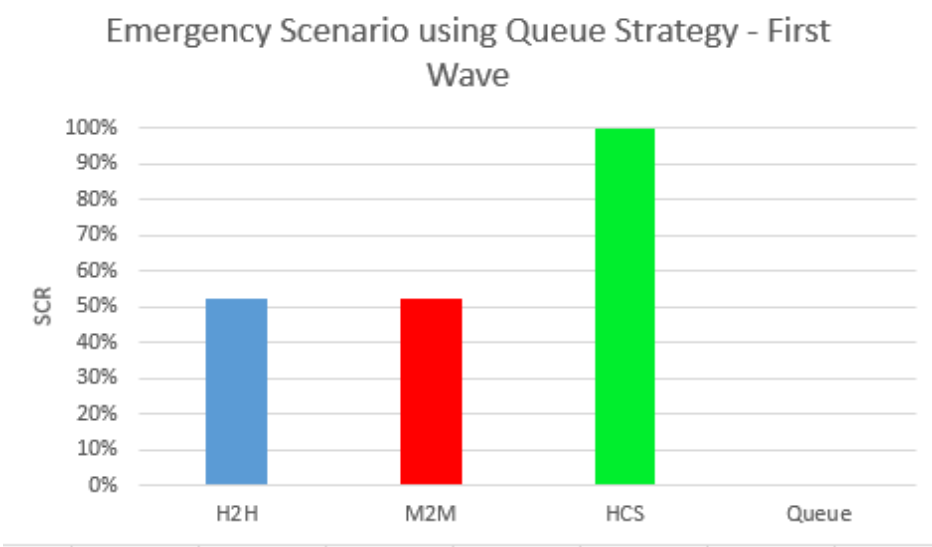

Figure 13. SCR of an emergency scenario using queue strategy (first storm wave).

According to the results shown in Figure 13, HCS traffic with the highest priority was fully served in the LTEA network with six PRBs, while the remaining bandwidth was divided between $\mathrm{H} 2 \mathrm{H}$ and $\mathrm{M} 2 \mathrm{M}$ traffic since they shared the same priority level. Moreover, as seen in the queue, 1,430 H2H and M2M traffic arrivals were pushed to the queue.

\section{Second Storm for Emergency Scenario Using Queue Strategy}

In the second wave, we assumed that, after one minute, a new wave or storm of emergency traffic had arrived as per the following parameters:

- An arrival rate of $\mathrm{H} 2 \mathrm{H}$ requests such that $\lambda_{\mathrm{H} 2 \mathrm{H}}=1$;

- An arrival rate of $\mathrm{M} 2 \mathrm{M}$ requests such that $\lambda_{\mathrm{M} 2 \mathrm{M}}=1$;

- An arrival rate of $\mathrm{HCS}$ requests such that $\lambda_{\mathrm{HCS}}=3$;

- An arrival rate of queue requests such that $\lambda_{\text {Queue }}=1.43$;

- $\mu_{\mathrm{H} 2 \mathrm{H}}=0.5, \mu_{\mathrm{M} 2 \mathrm{M}}=1, \mu_{\mathrm{HCS}}=1$, and $\mu_{\text {Queue }}=1$.

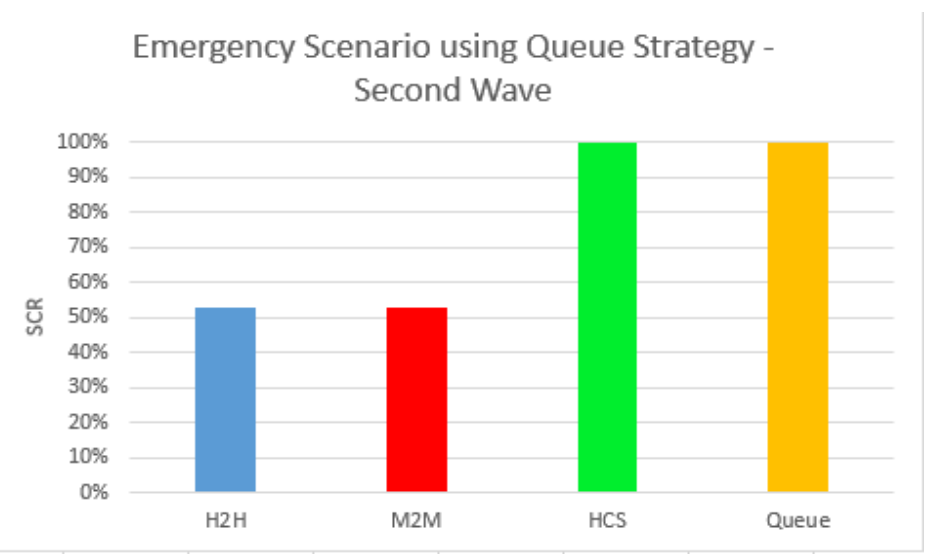

Figure 14. SCR of an emergency scenario using queue strategy (second storm wave). 
Figure 14 shows the results of the second storm wave. The highest priority was given to the queue to complete the arrival traffic that was pushed to the queue in the first wave of the storm. The second-highest priority was assigned to HCS traffic, while $\mathrm{H} 2 \mathrm{H}$ and M2M traffic had the same priority level. The SCR of the queue traffic was $100 \%$, and since the queue traffic was from the previous storm (in this case, $\mathrm{H} 2 \mathrm{H}$ and M2M traffic), we increased the SCR for $\mathrm{H} 2 \mathrm{H}$ and $\mathrm{M} 2 \mathrm{M}$ from $52 \%$ to $100 \%$ (from the previous wave).

Moreover, the overall delay time for a request in the queue was $0.05 \mathrm{~ms}(50 / 1000)$. It is found that, according to UCA OpenSG specifications, the maximum acceptable delay latency for a delay-tolerant application is $60 \mathrm{~s}$, for an average payload of $1.2 \mathrm{kB}$ and an arrival rate of six messages per day per device (Kumar et al., 2016). However, the acceptable delay latency for a delay-sensitive application is less than $3 \mathrm{~s}$, it shows a payload of less than $25 \mathrm{~B}$ and an arrival rate of 96 messages per day per device. Therefore, our delay latency is acceptable for both a delay-tolerant request and a delay-sensitive application since our requests are delayed for only $0.05 \mathrm{~ms}$ to be served in the second wave of the storm.

Furthermore, when we doubled the bandwidth, the SCR of all traffic was 100\%, the same as when we used the queue strategy. However, when we prioritized HCS traffic over all other traffic in such an emergency event, HCS traffic recorded $100 \%$, while $\mathrm{H} 2 \mathrm{H}$ and $\mathrm{M} 2 \mathrm{M}$ recorded $73 \%$. When we used an LTE-A and LTE-M hybrid network, HCS and $\mathrm{H} 2 \mathrm{H}$ traffic recorded $100 \%$, while M2M traffic recorded $70 \%$. In summary, all results show an improvement in the network's performance and QoS. However, the best results were achieved when we doubled the bandwidth and used queue modeling since the SCR maintained 100\%, and the QoS of H2H, M2M, and HCS traffic was not affected at all. On the other hand, doubling the bandwidth is not the optimal solution since it is costlier for the government to increase the bandwidth in every LTE-A network. A queue strategy is more efficient and less expensive, as shown in Figure 15.

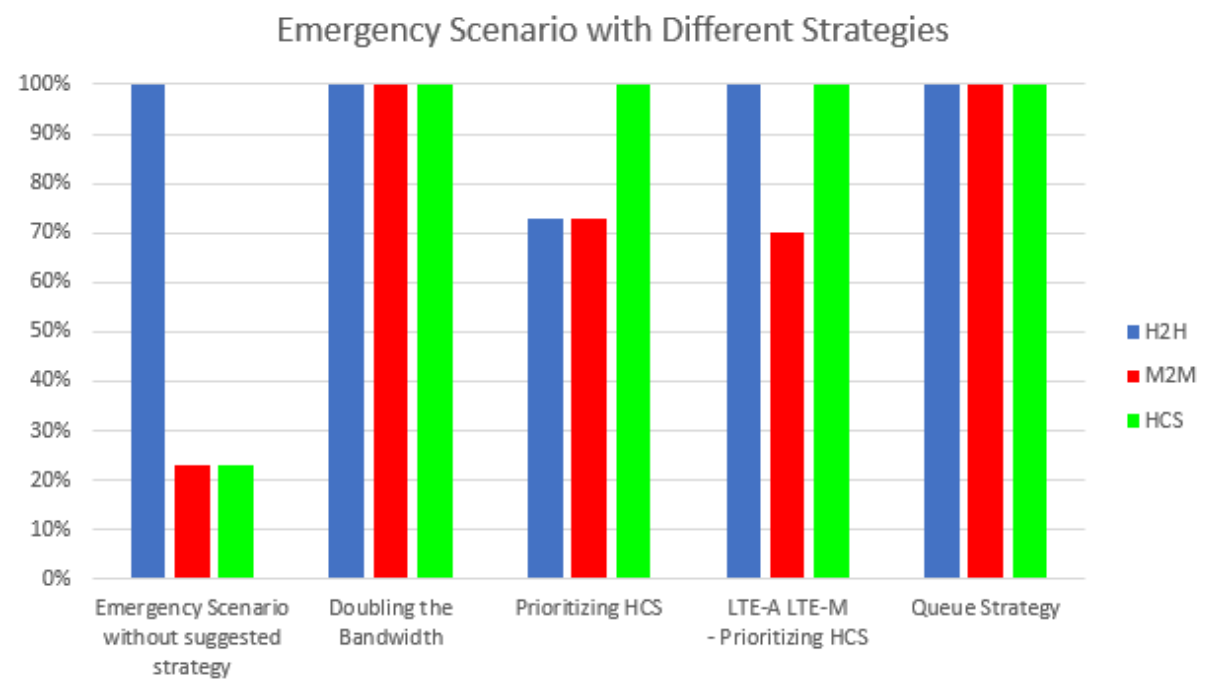

Figure 15. Emergency scenario with different strategies.

\section{Conclusion and Future Work}

In this area, many researchers have focused on addressing the coexistence of $\mathrm{H} 2 \mathrm{H}$ and M2M devices over LTE networks and the challenges faced by $\mathrm{M} 2 \mathrm{M}$ devices. The authors have proposed many solutions to this problem, such as giving priority to $\mathrm{H} 2 \mathrm{H}$ and M2M traffic or using the LTE-A and LTE-M networks for $\mathrm{H} 2 \mathrm{H}$ and M2M traffic, 
respectively. Others have worked on finding a solution to the bandwidth starvation problem. In our study, we improved the network to fulfill the needs of HCS devices to keep track of the health of people infected with contagious diseases. In addition, we proposed more than one suitable solution for the network overload and bandwidth starvation problems. The optimal solution was found when we maintained the QoS of all traffic and fulfilled our main target: to fulfill all HCS traffic in an emergency case without letting $\mathrm{H} 2 \mathrm{H}$ and M2M traffic impact it. In future work, we plan to assign different priorities in the queue for emergency events so that the HCS traffic pushed to the queue will be served first in the next storm before the $\mathrm{H} 2 \mathrm{H}$ and M2M requests. Furthermore, we can imagine a system with multiple queues where each queue is reserved for one type of traffic instead of sharing the same queue. This way, the QoS of each traffic type will not be affected in the event of a huge number of devices accessing the network.

\section{REFERENCES}

5G Global Launches \& Statistics. (n.d.). Future Networks. Retrieved May 14 2021, from https://www.gsma.com/futurenetworks/ip_services/understanding-5g/5g-innovation/

Bai, L., Yang, D., Wang, X., Tong, L., Zhu, X., Zhong, N., Bai, C., Powell, C. A., Chen, R., Zhou, J., Song, Y., Zhou, X., Zhu, H., Han, B., Li, Q., Shi, G., Li, S., Wang, C., Qiu, Z., ... Tan, F. (2020). Chinese experts' consensus on the Internet of Things-aided diagnosis and treatment of coronavirus disease 2019 (COVID-19). Clinical EHealth, 3, 7-15. https://doi.org/10.1016/j.ceh.2020.03.001

Bajaj, R. K., Rao, M., \& Agrawal, H. (n.d.). Internet Of Things (IoT) In The Smart Automotive Sector: A Review. Internet Of Things, 9.

Chen, X., Ma, M., \& Liu, A. (2018). Dynamic power management and adaptive packet size selection for IoT in e-Healthcare. Computers \& Electrical Engineering, 65, 357-375. https://doi.org/10.1016/j.compeleceng.2017.06.010

Coronavirus disease (COVID-19)—Pandemic. (n.d.). Retrieved 14 May 2021, from https://www.who.int/emergencies/diseases/novel-coronavirus-2019

EI Fawal, A. H., Najem, M., Mansour, A., Le Roy, F., \& Le Jeune, D. (2018). CTMC modelling for H2H/M2M coexistence in LTE-A/LTE-M networks. The Journal of Engineering, 2018(12), 1954-1962. https://doi.org/10.1049/joe.2018.5042

Harb, H., Mroue, H., Mansour, A., Nasser, A., \& Motta Cruz, E. (2020). A Hadoop-Based Platform for Patient Classification and Disease Diagnosis in Healthcare Applications. Sensors, 20(7), 1931. https://doi.org/10.3390/s20071931

Ikpehai, A., Adebisi, B., Rabie, K. M., Anoh, K., Ande, R. E., Hammoudeh, M., Gacanin, H., \& Mbanaso, U. M. (2019). Low-Power Wide Area Network Technologies for Internet-of-Things: A Comparative Review. IEEE Internet of Things Journal, 6(2), 2225-2240. https://doi.org/10.1109/JIOT.2018.2883728

Kumar, A., Abdelhadi, A., \& Clancy, C. (2016). A Delay-Optimal Packet Scheduler for M2M Uplink. ArXiv:1606.06794 [Cs, Math]. http://arxiv.org/abs/1606.06794

Mehmood, Y., Ahmad, F., Yaqoob, I., Adnane, A., Imran, M., \& Guizani, S. (2017). Internet-of-ThingsBased Smart Cities: Recent Advances and Challenges. IEEE Communications Magazine, 55(9), 16-24. https://doi.org/10.1109/MCOM.2017.1600514

Mutlag, A. A., Abd Ghani, M. K., Arunkumar, N., Mohammed, M. A., \& Mohd, O. (2019). Enabling technologies for fog computing in healthcare IoT systems. Future Generation Computer Systems, 90, 6278. https://doi.org/10.1016/j.future.2018.07.049 
Outay, F., Yasar, A.-U.-H., \& Shakshuki, E. (Eds.). (2020). Global Advancements in Connected and Intelligent Mobility: Emerging Research and Opportunities. IGI Global. https://oi.org/10.4018/978-15225-9019-4

Powell, A., \& University, H. (n.d.). Researchers develop smart tattoos for health monitoring. Retrieved May 14 2021, from https://phys.org/news/2017-09-smart-tattoos-health.html

Saeed, N., \& Alouini, M.-S. (n.d.). When Wireless Communication Faces COVID-19: Combating the Pandemic and Saving the Economy. 12.

Song, Y., Jiang, J., Wang, X., Yang, D., \& Bai, C. (2020). Prospect and application of Internet of Things technology for prevention of SARIs. Clinical EHealth, 3, 1-4. https://doi.org/10.1016/j.ceh.2020.02.001

Ullah, H., Gopalakrishnan Nair, N., Moore, A., Nugent, C., Muschamp, P., \& Cuevas, M. (2019). 5G Communication: An Overview of Vehicle-to-Everything, Drones, and Healthcare Use-Cases. IEEE Access, 7, 37251-37268. https://doi.org/10.1109/ACCESS.2019.2905347

Zhong, L., Mu, L., Li, J., Wang, J., Yin, Z., \& Liu, D. (2020). Early Prediction of the 2019 Novel Coronavirus Outbreak in the Mainland China Based on Simple Mathematical Model. IEEE Access, 8, 51761-51769. https://doi.org/10.1109/ACCESS.2020.2979599 